\title{
Recurrent Aspergillus endocarditis in an immunocompetent patient: challenges in diagnosis and management
}

Yuan Ye Beh* Medical student, University of Aberdeen, Grace Li Hsien Lim Medical student, University of Aberdeen, Andrew

Stewart, Cardiology, Aberdeen Royal Infirmary

*Corresponding author: yuanye.beh.14@aberdeen.ac.uk

ABSTRACT

We describe an unusual case of recurrent Aspergillus endocarditis in an immunocompetent 64-year-old lady. Four weeks after an aortic valve replacement surgery for severe stenosis of a bicuspid aortic valve, she presented with an inferior ST-elevation myocardial infarction. Coronary angiography demonstrated compromise of the ostium of the right coronary artery, which was successfully treated by primary angioplasty and stenting. Six weeks later, she suffered from a subarachnoid haemorrhage secondary to a mycotic aneurysm. A transoesphageal echocardiogram suggested a large aortic root vegetation. She underwent urgent aortic root replacement with removal of the vegetation, which was subsequently confirmed to be caused by Aspergillus. She was discharged on long-term anti-fungal medication (voriconazole) which she did not adhere to. Several years later, she presented with non-specific symptoms and was ultimately diagnosed with a recurrence of Aspergillus endocarditis. This case illustrates one of the many nonspecific ways Aspergillus endocarditis can present. A high index of suspicion can prevent significant life-threatening complications.

\section{BACKGROUND}

Fungal endocarditis is uncommon, representing less than $2 \%$ of all cases of endocarditis. Of this $2 \%$, only $20-25 \%$ are represented by Aspergillus. ${ }^{1}$ It usually affects patients who are immunocompromised ${ }^{1}$ Cardiac fungal infections carry a high mortality. The diagnosis is challenging as the symptoms are nonspecific and blood cultures are usually negative. ${ }^{2} \mathrm{~A}$ timely diagnosis is important, as complications of untreated endocarditis include life-threatening haemodynamic disturbances, peripheral septic embolism, and systemic sepsis. Patients with Aspergillus endocarditis are usually recommended to have surgical resection and then continue on long-term anti-fungal therapy. ${ }^{3}$

This unfortunate patient described here suffered many complications following aortic valve replacement, all of which were ultimately related to Aspergillus endocarditis: a myocardial infarction, an intracranial bleed from a mycotic aneurysm, an aortic root replacement and, finally, a recurrence of the infection.

\section{CASE PRESENTATION}

We describe a case of an immunocompetent 64-yearold lady who underwent aortic valve replacement for severe stenosis of a bicuspid aortic valve. She had a history of recurrent urinary tract infections but no other major health problems. Her valve surgery was uneventful and she made a good initial recovery.

Despite normal coronary arteries on her preoperative angiogram, the patient presented with an inferior STelevation myocardial infarction four weeks after her surgery. She was taken directly to the cardiac catheterization laboratory for primary percutaneous coronary intervention. There was a severe compromise of the ostium of her right coronary artery, which was treated by angioplasty and stenting. The angiogram also demonstrated a slight abnormality in appearance 
of the aortic root. A transoesphageal echocardiogram (TOE) suggested a localized dissection of the aortic root, which was felt to be related to either the coronary angiogram procedure or to her recent surgery. She remained well and was treated conservatively. Six weeks later, she suffered a subarachnoid haemorrhage and underwent cerebral angiography. This demonstrated a mycotic aneurysm, which was successfully coiled. A further TOE was performed which, on this occasion, demonstrated a large vegetation attached to her aortic root. The prosthetic valve appeared unaffected.

The affected aortic root was resected and replaced with an aortic homograft. Microbiological analysis of the aortic root sample showed a positive result for Aspergillus. The recently replaced valve was spared. She was discharged well on long-term anti-fungal therapy (voriconazole), which she did not adhere to.

Three years after her first operation, she presented with confusion, fever, word finding difficulties, urinary incontinence, and leg weakness. There were no clinical stigmata of infective endocarditis.

\section{INVESTIGATIONS}

On admission, an urgent computed tomography (CT) scan of her head was ordered along with a series of other investigations. The results are listed in Table 1 .

Table 1. Investigations and their findings

\begin{tabular}{|c|c|}
\hline INVESTIGATIONS & FINDINGS \\
\hline CT head & Normal \\
\hline Urinalysis & $\begin{array}{c}\text { Leucocyte ++ } \\
\text { Blood ++++ } \\
\text { Protein }+ \\
\text { Nitrites }+++\end{array}$ \\
\hline $\begin{array}{l}\text { Mid-stream sample } \\
\text { urine (MSSU) }\end{array}$ & Escherichia coli \\
\hline $\begin{array}{c}\text { Transthoracic } \\
\text { echocardiography }\end{array}$ & $\begin{array}{l}\text { Mild mitral and tricuspid } \\
\text { valve regurgitation } \\
\text { Satisfactory function of } \\
\text { prosthetic aortic valve } \\
\text { No evidence of vegetation }\end{array}$ \\
\hline
\end{tabular}

\section{DIFFERENTIAL DIAGNOSIS}

i) Urinary tract infection (UTI)

The positive urinalysis result and the isolation of $\mathrm{E}$. coli from MSSU confirmed the presence of a UTI. Indeed, the patient had a history of recurrent UTIs.

ii) Acute stroke/transient ischemic attack due to septic emboli from infective endocarditis

As she presented with some neurological signs, a diagnosis of stroke was considered. However, as her CT head scan was normal, it was decided at the time to monitor her clinically with a plan for discharge if she remains stable.

\section{TREATMENT}

She was initially treated empirically for urinary sepsis with intravenous (IV) amoxicillin plus gentamicin. This regimen was changed to IV piperacillin/tazobactam and oral co-amoxiclav after microbiology report isolated E. coli sensitive to piperacillin/tazobactam from her urine.

\section{OUTCOME AND FOLLOW UP}

Her pyrexia persisted for two weeks despite antibiotic treatment. In view of this, a TOE was arranged to definitively exclude a recurrence of Aspergillus endocarditis. The TOE showed a large mobile mass attached to the wall of proximal ascending aorta (Figure 1).

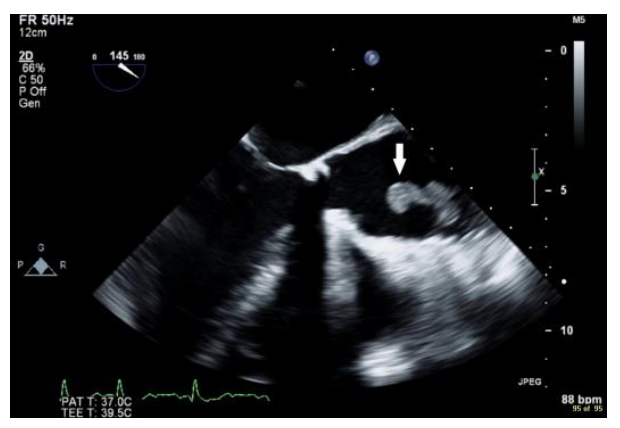

Figure 1. Large vegetation in ascending aorta

Whilst the results of her blood cultures and Aspergillus polymerase chain reaction were negative, her plasma beta-glutan concentration (a marker of fungaemia) was 
markedly raised in two consecutive samples (>500 $\mathrm{pg} / \mathrm{mL}$ ). These results, considered along with her clinical picture, past medical history (Aspergillus endocarditis, aortic valve replacement, and aortic root replacement), and previous non-adherence to longterm anti-fungal therapy, established the diagnosis of recurrent Aspergillus endocarditis.

A cardiothoracic opinion was sought due to the large size of the vegetation and the fact that she suffered from recurrent transient neurological symptoms (which were thought to be due to recurrent cerebral microemboli). Although she was offered further surgery to resect the large aortic vegetation, both the patient and her family were reluctant to consider a third major cardiac operation. She remained well, her temperature and inflammatory markers settled, and she was ultimately discharged to supported accommodation on long-term oral voriconazole.

\section{DISCUSSION}

This case demonstrates a case of fungal endocarditis that led to a series of complications in a nonimmunocompromised patient.

When the lady presented with an acute inferior myocardial infarction soon after aortic valve surgery, less common causes had to be considered, as a preoperative angiogram had shown normal coronaries arteries. It was noted in a subsequent angiogram that her aortic root looked abnormal, but this was felt to be either related to disruption from her operation or to be caused during the angioplasty procedure. In retrospect, however, this appearance was almost certainly due to an aortic root vegetation. She had no clinical features to suggest endocarditis at that time. However, had this been recognized, the subsequent clinical course could potentially have been different.

Aspergillus endocarditis is uncommon and requires a very high index of suspicion to diagnose. Blood cultures are frequently negative. ${ }^{2}$ Measurement of plasma beta-glucan can be a very sensitive test of fungaemia with a cut-off value of $80 \mathrm{pg} / \mathrm{mL}$. ${ }^{1}$ Though the diagnostic yield of this test for Aspergillus endocarditis remains poorly documented, ${ }^{1}$ a previous history of Aspergillus endocarditis coupled with grossly elevated beta-glucan $(>500 \mathrm{pg} / \mathrm{mL})$ in two samples rendered a recurrence most likely.

Previous cardiac surgery is a recognized risk factor for fungal endocarditis and is the most likely cause of Aspegillus infection in this patient. The modified Duke's criteria for the diagnosis of infective endocarditis were met in this case with a vegetation demonstrated on TOE (major criterion), fever (minor criterion), vascular phenomena (minor criterion) and a predisposing heart condition (minor criterion). ${ }^{4}$

Following her aortic root replacement surgery, the plan was for her to remain on long-term voriconazole. However, the patient elected to discontinue this after only seventeen weeks. This was likely contributory to the recurrence of her Aspergillus fungaemia. It could also be argued that her aortic valve should have been replaced at the time of the aortic root replacement in case it was infected. However, it was the opinion of the surgeon at that time that it was clear of infection.

In a review looking into published case reports on Aspergillus endocarditis from 1950 to 2010, only 4\% of cases declared treatment success without surgical intervention.5 Up to $60 \%$ of the patients included in the same review were immunosuppressed.5 However, being immunocompetent does not exclude a diagnosis of Aspergillus endocarditis, as demonstrated in this case as well as in two other case reports published in 2005 and 2015 respectively. As with our patient in this case, both case reports described incidences of Aspergillus endocarditis in immunocompetent patients after cardiac surgery (aortic valve replacement and aortic root replacement). ${ }^{2,6}$ 


\section{PATIENT'S PERSPECTIVES}

"Our lives have been changed for the worst... she's not mum anymore... This affects all of us [referring to siblings]... Mum feels sorry for herself."

- script adapted from verbal response given by patient's daughter.

\section{LEARNING POINTS}

$>$ A very high index of suspicion is required to diagnose Aspergillus endocarditis.

$>$ Aspergillus endocarditis can occur in patients who are immunocompetent, particularly after cardiac surgery.

$>$ Aspergillus endocarditis can be associated with significant life-threatening complications.

$>$ Use of long-term antifungal therapy and appropriate follow-up is essential in minimizing the risk of recurrence of fungal endocarditis.

$>$ Patients with features of endocarditis, who are unresponsive to antibiotic treatment, should prompt investigation for rarer causes such as fungal endocarditis due to Aspergillus.

\section{REFERENCES}

1. Tattevin P, Revest M, Lefort A, Michelet C, Lortholary O. Fungal endocarditis: current challenges. Int J Antimicrob Agents. 2014;44(4):290-4. DOI: 10.1016/j.ijantimicag.2014.07.003.

2. Bapat V, Young C. Aspergillus endocarditis presenting as massive peripheral embolism following open heart surgery. EJVES Extra. 2005;10(1):27-9. DOI: 10.1016/j.ejvsextra.2005.04.014.

3. Gould FK, Denning DW, Elliott TS, Foweraker J, Perry JD, Prendergast BD, et al. Guidelines for the diagnosis and antibiotic treatment of endocarditis in adults: a report of the Working Party of the British Society for Antimicrobial Chemotherapy. J Antimicrob Chemother. 2012;67(2):269-89. DOI: Int J Antimicrob Agents. 2014;44(4):290-4. DOI: 10.1016/j.ijantimicag.2014.07.003.

2. Bapat V, Young C. Aspergillus endocarditis presenting as massive peripheral embolism following open heart surgery. EJVES Extra. 2005;10(1):27-9. DOI: 10.1016/j.ejvsextra.2005.04.014.

3. Gould FK, Denning DW, Elliott TS, Foweraker J, Perry JD, Prendergast BD, et al. Guidelines for the diagnosis and antibiotic treatment of endocarditis in adults: a report of the Working Party of the British Society for Antimicrobial Chemotherapy. J Antimicrob Chemother. 2012;67(2):269-89. DOI: 10.1093/jac/dkr450.

4. Habib G, Lancellotti P, Antunes MJ, Bongiorni MG, Casalta JP, Del Zotti F, et al. 2015 ESC Guidelines for the management of infective endocarditis. Eur Heart J. 2015;36(44):3075-128. DOI: 10.1093/eurheartj/ehv319.

5. Kalokhe AS, Rouphael N, El Chami MF, Workowski KA, Ganesh G, Jacob JT. Aspergillus endocarditis: a review of the literature. Int J Infect Dis. 2010;14(12):e1040-7. DOI: 10.1016/j.ijid.2010.08.005.

6. Hussein N, Qamar S, Abid Q. Systemic aspergilloma post aortic root surgery following coronary artery stenting: diagnostic and management dilemma. BMJ Case Rep. 2015;2015. DOI: 10.1136/bcr-2014-207702. 